\title{
An essential review of Singapore's response to out-of-hospital cardiac arrests: improvements over a ten-year period
}

\author{
Alexander $E \underline{\text { White }}^{1,2}$, JD, MPH, Andrew FW $\underline{\mathrm{Ho}}^{2,3}, \mathrm{MBBS}, \mathrm{MPH}$, Nur Shahidah ${ }^{3}$, BA, Nurul Asyikin ${ }^{1}, \mathrm{BSc}$, Le Xuan Liew ${ }^{1}$, BSc, \\ Pin Pin Pek ${ }^{2,3}$, MPH, Jade PH Kua ${ }^{4}$, MBBs, MRCs, Michael YC Chia ${ }^{5}$, MBBS, Yih Yng Ng ${ }^{5,6}, \mathrm{MBBS}, \mathrm{MPH}$, \\ Shalini Arulanandam ${ }^{7}$, MMed, MBA, Sieu-Hon Benjamin Leong ${ }^{8}$, MBBS, MRCSEd, Marcus EH Ong $^{3,9}$, MBBS, MPH
}

\begin{abstract}
Care for patients who experience out-of-hospital cardiac arrest (OHCA) has rapidly evolved in the past decade. Increased sophistication of care in the community, emergency medical services (EMS) and hospital setting is associated with improved patient-centred outcomes. Notably, Utstein survival doubled from $11.6 \%$ to $23.1 \%$ between 2011 and 2016. These achievements involved collaboration between policymakers, clinicians and researchers, and were made possible by a strategic interplay of policy, research and implementation. We review the development and current state of OHCA in Singapore using primary population-based data from the Pan-Asian Resuscitation Outcomes Study and an unstructured search of research databases. We discuss the roles of important milestones in policy, community, dispatch, EMS and hospital interventions. Finally, we relate these interventions to relevant processes and outcomes, such as the relationship between the strategic implementation of bystander cardiopulmonary resuscitation and placement of automated external defibrillator with return of spontaneous circulation, survival to discharge and survival with favourable neurological outcomes.
\end{abstract}

Keywords: CPR+AED, dispatchers, feedback, out-of-hospital cardiac arrest, smartphone applications

\section{INTRODUCTION}

To bring about substantive, long-standing improvements in healthcare, a national- and systems-level approach should ideally be followed. However, many countries do not have this luxury and should aspire to achieve a high level of cooperation and buy-in regardless of the time required. In short, this was how Singapore approached the challenge to improve the survival rates of patients who experience out-of-hospital cardiac arrest (OHCA).

Our journey began with a group of idealistic emergency physicians who observed how other systems worldwide were achieving survival rates about three times higher than those observed in Singapore. ${ }^{(1)}$ Singapore had only single-digit survival rates, topping at $3.5 \%$ by the end of 2011. ${ }^{(2,3)}$ By 2012, the implementation of new initiatives and escalating support led to a gradual increase in the survival rate for OHCA. After years of data collection, evidence gathering and planning, researchers convinced the Ministry of Health $(\mathrm{MOH})$, Singapore that better outcomes could be achieved. From 2011 to 2018, Singapore witnessed a rise in overall survival-to-discharge rate from $3.5 \%$ to $5.9 \%$ and increased Utstein (witnessed, shockable) survival rate from $11.6 \%$ to $25.9 \%$ among patients with $\mathrm{OHCA} .{ }^{(4)}$

\section{OHCA REGISTRY IN SINGAPORE Cardiac Arrest and Resuscitation Epidemiology}

The journey of OHCA research in Singapore began in 2002 with the Cardiac Arrest and Resuscitation Epidemiology (CARE) Phase I project. The project aimed to describe the epidemiology of OHCA in Singapore using data collected between 1 October 2001 and 30 April 2002. Based on data (548 cases) collected over seven months, a $20.6 \%$ rate of bystander cardiopulmonary resuscitation (CPR) and an overall 2.0\% survival-to-discharge rate was reported. ${ }^{(5)}$ Results from the CARE I project provided preliminary insights regarding the incidence of OHCA in Singapore and presented an opportunity to implement subsequent phases such as CARE II to further improve and strengthen the chain of survival (a concept depicting the various components of a systematic approach to OHCA response, leading to optimal patient outcomes). ${ }^{(6)}$ CARE Phase II sought to evaluate the benefits of introducing intravenous epinephrine in the prehospital setting. Although the study did not show significant survival benefit in patients, ${ }^{(7)}$ it captured the interest of emergency care providers in Singapore and fuelled further research in the field of OHCA and prehospital care.

\section{Pan-Asian Resuscitation Outcomes Study Clinical Research Network}

In 2010, the Pan-Asian Resuscitation Outcomes Study Clinical Research Network (PAROS CRN), led by Singapore, was developed as a platform to improve outcomes in prehospital and emergency care across the Asia-Pacific by promoting high-quality research in resuscitation. Comprising dedicated emergency physicians and prehospital care providers from across the AsiaPacific, the CRN has grown from seven to 13 countries, with more

\footnotetext{
${ }^{1}$ Unit for Prehospital Emergency Care, Singapore General Hospital, ${ }^{2}$ Pre-hospital and Emergency Research Centre, Duke-NUS Medical School, ${ }^{3}$ Department of Emergency Medicine, Singapore General Hospital, ${ }^{4}$ Emergency Department, Woodlands Health Campus, ${ }^{5}$ Emergency Department, Tan Tock Seng Hospital, ${ }^{6} \mathrm{Home}$ Team Medical Services Division, Ministry of Home Affairs, ${ }^{7}$ Emergency Medical Services Department, Singapore Civil Defence Force, ${ }^{8}$ Emergency Medicine Department, National University Hospital, ${ }^{9}$ Health Services and Systems Research, Duke-NUS Medical School, Singapore

Correspondence: Mr Alexander E White, Research and Policy Lead, Unit for Prehospital Emergency Care, c/o Singapore General Hospital, Outram Road, Singapore 169608. alexander.elgin.white@upec.sg
} 
than 30 participating sites to date. A new set of data elements, including those contained in the Utstein reporting template along with a data taxonomy, was included with consensus from PAROS executive members. ${ }^{\left({ }^{8}\right)}$

\section{PAROS collaboration}

The PAROS CRN's association with the Cardiac Arrest Registry to Enhance Survival (CARES) started with its collaboration with the Centers for Disease Control and Prevention, Atlanta, USA. CARES has supported PAROS in developing and managing an electronic data capture system known as ePAROS, which is available free of charge to PAROS members. The ePAROS software collects data about OHCA. PAROS is also supported by the Singapore Clinical Research Institute. This collaboration also involves reconciliation of data elements for potential CARES-PAROS projects, which helps to establish international benchmarks for the outcomes of $\mathrm{OHCA}$ to improve patient survival.

\section{National population health survey}

A national population health survey on the knowledge, attitudes and practices of Singapore residents towards life-saving skills was conducted from November 2009 to March 2010. A total of 7,840 household addresses were randomly sampled and selected for this survey. The response rate was $65.2 \%$ (4,192 respondents), and the survey results showed that a high proportion of respondents believed that adults should be trained in first aid (89.1\%) and CPR (82.6\%). ${ }^{(9)}$ The survey also found that the proportion of those who had 'ever been' trained in these skills was very low, which was a significant finding of the study. ${ }^{(9)}$ Data collected and contributed by the Singapore cohort of PAROS can be used to identify and support areas of need such as community training in CPR and automated external defibrillator (AED) skills. This will, in turn, improve community responsiveness and confidence in rendering assistance for cardiac arrest incidents, likely leading to improved bystander CPR rates, AED application and, eventually, better survival outcomes. Data is critical to garner decision-maker support and funding to implement changes or interventions. Galvanising change-makers to get work done at the grassroots level is the next essential step.

\section{DEVELOPING ALLIES: MANY HANDS MAKE LIGHT WORK \\ First five years}

Between 2012 and 2017, efforts to increase survival from OHCA were guided by our first five-year strategic plan, which served as our blueprint to address prehospital emergency care (PEC) objectives, including OHCA. A unit for PEC (UPEC), comprising part-time emergency medical services (EMS) physicians, paramedics, nurses and executive staff hosted under Singapore General Hospital, was set up by the $\mathrm{MOH}$ to support the implementation of this five-year plan. Fig. 1 shows the timeline of key initiatives that contributed to improvements in the prehospital setting in Singapore.

Collaboration with the Singapore Civil Defence Force (SCDF), $\mathrm{MOH}$ and UPEC was essential for the success of this plan. The
SCDF runs fire-based EMS in Singapore. The $\mathrm{MOH}$ funded the establishment of UPEC and facilitates the passage of any policy or protocol changes. UPEC functions in the wider domain of prehospital emergency care; however, its early role in convening and marshalling the input of thought leaders was a major reason for the focus on OHCA and the eventual progress made. The benefits of convening stakeholders and thought leaders should not be overlooked. An emergency physician from each of the public hospitals was part of the UPEC setup. The Chief Medical Officer of SCDF and emergency medical dispatch nurses who handle cardiac arrest calls were also participants. This collaboration allowed ideas, problems and solutions to be discussed by the people who could implement the required changes. Monthly stakeholder group meetings were a routine, and these were enriched with presentations by invited academia, stakeholders in the industry and other partners, who presented their innovations or proposals for collaboration.

The first five-year strategic plan kickstarted UPEC and its subsequent efforts. The second five-year plan (2017-2022) was important because it helped to solidify the cooperation required across organisations. Group leaders from SCDF, MOH and UPEC were tasked with reporting the progress of the objectives from the previous five-year plan. Then, a larger group from these organisations convened at a retreat, where workgroups were formed for each strategic domain. Using a consensus-based approach, each workgroup developed its own assessment of the previous goals and set new ones. In the weeks after the retreat, the UPEC staff collated the finalised workgroup priorities into a working document.

In January 2016, leaders from MOH, SCDF and UPEC attended a half-day stakeholder meeting, where they assessed which of the goals were practical and achievable within the next five years, and garnered management-level support among themselves. Some goals were removed and others were refined. Overall, the attendees achieved consensus on the second five-year plan of the UPEC. Based on this strategic plan, the UPEC sought multi-ministry approval and funding to carry out its objectives.

\section{COMMUNITY INTERVENTIONS}

An innovative community-based training programme called 'Dispatcher-Assisted first REsponder (DARE)' was launched in 2014. This 45-minute programme, which is video-guided along with hands-on training, teaches participants how to save lives through simplified compression-only CPR and the use of an AED. ${ }^{(10)}$ Studies have shown that the compression-only approach yielded equal or slightly better outcomes for adults with OHCA and helped reduce barriers and delays to CPR. ${ }^{(11,12)}$ The learning objectives of DARE are: immediately contact ' 995 ' for an ambulance and stay on the line with the medical dispatcher; perform chest compressions on the centre of the chest by pushing hard and fast; and inform someone to get the AED and then use it. ${ }^{(10)}$ First piloted in schools, ${ }^{(10)}$ DARE has, over the years, spread across the nation, including business sites, places of worship and community clubs.

To assist with chest compressions, the CPRcard ${ }^{\mathrm{TM}}$, another innovative tool that complements the DARE programme, was 


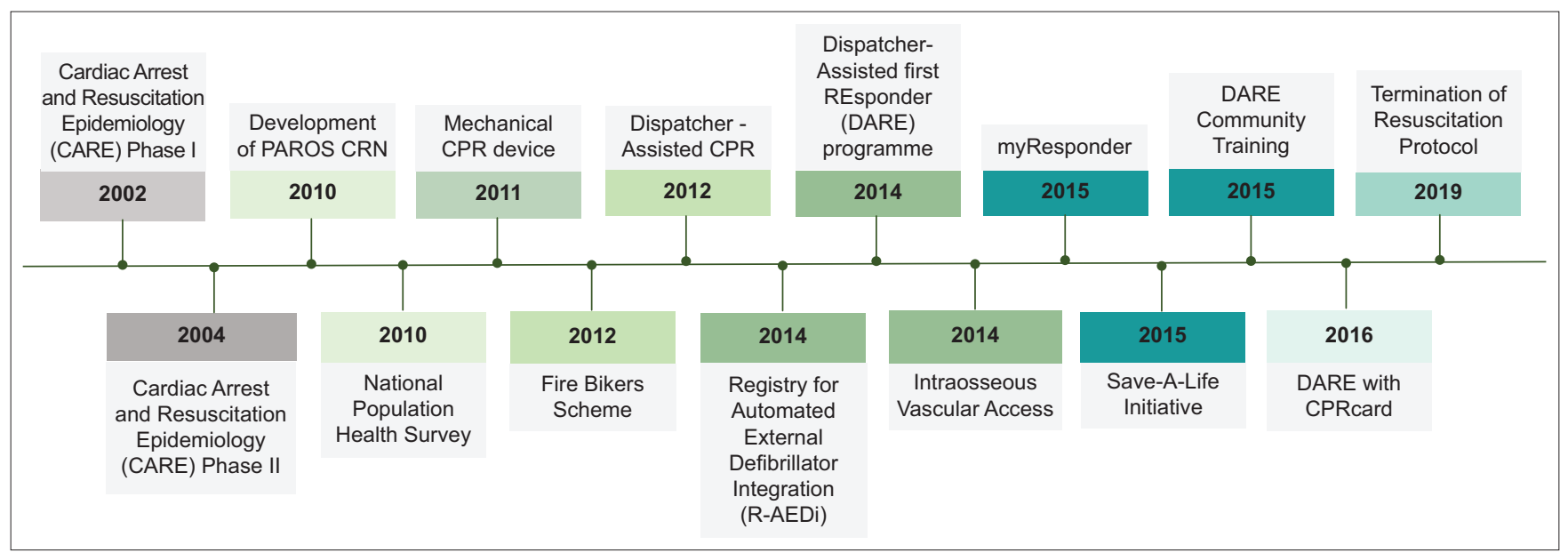

Fig. 1 Chart shows the timeline of key prehospital initiatives. CPR: cardiopulmonary resuscitation; CRN: clinical research network; PAROS CRN: Pan-Asian Resuscitation Outcomes Study Clinical Research Network

initiated. The CPRcard is a novel, credit card-sized feedback device that provides visual assistance to achieve proper depth and rate of chest compressions. ${ }^{(13)}$ It is part of a research study in collaboration with Laerdal Medical (Norway) to evaluate whether the DARE programme and CPRcard training would bring about a change in the attitude and confidence of the public in saving lives and whether they lead to any improvements in bystander response rates and victim survival. Participants receive a CPRcard at the start of their training and use it during their compression practice. The compression data is stored in the CPRcard and extracted at the end of the training. Pre- and post-training surveys are part of the training experience for these participants. This allows researchers to observe how participants viewed CPR and AED use before and after the training. One study that compared the quality of chest compressions with and without the use of the CPRcard showed that better-quality compressions were delivered by the participants with the CPRcard feedback compared with those delivered without feedback (net compression rate of 100-120/min, depth $\geq 5 \mathrm{~cm}$, $36 \%$ and $4.0 \%$, respectively; $p=0.022) .{ }^{(13)}$ Moreover, most of the participants responded positively, stating that using the feedback device increased their confidence in performing chest compressions (median score $2.7 \pm 2.3$, where 1 is 'very confident' and 10 is 'not confident'). ${ }^{(13)}$ This confidence would presumably extend to real cases.

At the end of the training, participants are encouraged to download myResponder, ${ }^{(14)}$ a smartphone application created by the SCDF. Such smartphone technology, in general, has the potential to reach out to the masses and get 'Good Samaritans' to help victims before the ambulance arrives, significantly increasing bystander CPR rates. ${ }^{(14-17)}$ The key features of the myResponder app are: (a) '995' call button with enabled GPS to detect the location of the incident; (b) AED registry for users to locate the nearest AED; and (c) capability to alert active responders who are app users to nearby OHCA cases occurring within $400 \mathrm{~m}$ of their location. ${ }^{(14)}$ As the CPRcards are also intended for use in real emergency cases, these CPRcard-trained residents can accept the app alert, attend to the case and use the CPRcard on a victim of OHCA before the ambulance arrives. Typically, CPRcards are used in real cases by myResponder app users. Since the launch of the myResponder app, we have observed an increasing proportion of cases where myResponders have accepted the alerts and arrived at the scene. In 2019, 45.8\% of myResponders accepted the notifications and $24.1 \%$ arrived at the scene. ${ }^{(14)}$ Of these, $43 \%$ arrived before an ambulance did. ${ }^{(14)}$ The last measure is an indicator of advanced public response to OHCA, which we hope will continually improve.

\section{EMERGENCY MEDICAL SERVICES AND EMERGENCY DEPARTMENTS}

The transformation of OHCA care in prehospital and hospital settings took place over a decade. From 2011, mechanical CPR devices were deployed in SCDF ambulances, initially as a pragmatic, cluster-randomised, pre-hospital trial comparing LUCAS 2 (Physio-Control, Redmond, WA, USA) with manual CPR and subsequently, as standard care. This study (MECCA trial), which was conducted between 2011 and 2012, has previously been described. ${ }^{(18)}$ Subsequently, LUCAS 2 was rolled out to all ambulances. In 2012, Singapore participated in a comprehensive dispatcher-assisted CPR (DA-CPR) implementation package as part of the PAROS Phase II study. It comprised dispatcher training focused on protocolised communication and persuasion, review of audio recordings of all OHCA calls, feedback to dispatchers and public education. ${ }^{(19)}$ After a planned six-month 'run-in' period, all dispatchers were able to provide DA-CPR consistently. ${ }^{20,21)}$

In 2014, intraosseous access was introduced to ambulances as part of a cluster-randomised prehospital trial. This was used in OHCA cases for administration of adrenaline after intravenous access attempts had failed. In 2019, the advanced life support termination of resuscitation protocol was adopted across Singapore. While data collection is ongoing, a Markov model simulation based on the data collected so far indicated reduced healthcare costs and fewer non-beneficial hospital admissions. ${ }^{(22)}$ Over the years, advanced hospital-based interventions, including targeted temperature management and urgent coronary angiography, have been adopted in varying degrees across Singapore. ${ }^{(23)}$ 
Table I. Recommended interventions for response to out-of-hospital cardiac arrest (OHCA).

\begin{tabular}{|c|c|c|c|}
\hline Institution & Community & EMS/ambulance & $\begin{array}{l}\text { Hospital/Emergency } \\
\text { Department }\end{array}$ \\
\hline $\begin{array}{l}\text { - Be it research, academic } \\
\text { medical institutions or } \\
\text { ministries of health, any } \\
\text { initiative must begin with } \\
\text { collecting data and taking } \\
\text { a measure of the baseline. } \\
\text { - Hospitals should } \\
\text { collaborate in achieving } \\
\text { consensus on what data } \\
\text { points will be collected } \\
\text { and gathered into a } \\
\text { centralised repository. }\end{array}$ & $\begin{array}{l}\text { - A basic level of CPR and AED skills } \\
\text { training should be implemented } \\
\text { widely through various means } \\
\text { and leverage technology if in- } \\
\text { person training is impractical. } \\
\text { - Certified CPR/AED/first aid } \\
\text { courses should be adequately } \\
\text { available, as required. } \\
\text { - Nonconventional means of } \\
\text { teaching should be considered to } \\
\text { reach the greatest numbers. } \\
\text { - Quality of training should remain } \\
\text { a goal but may come with time. } \\
\text { Awareness of what to look } \\
\text { for and what to do may be a } \\
\text { reasonable starting point. } \\
\text { - Public AED installations and } \\
\text { accessibility is another important } \\
\text { component because of the } \\
\text { important role AEDs have in } \\
\text { treating patients with OHCA with } \\
\text { shockable rhythm. } \\
\text { - CPR/AED training as a } \\
\text { requirement to get/renew } \\
\text { driver's licence is also a common } \\
\text { approach. } \\
\text { - School-based training } \\
\text { requirements as part of physical } \\
\text { education is a popular means of } \\
\text { training. }\end{array}$ & $\begin{array}{l}\text { - Gather data and engage in continuous } \\
\text { improvement. Share data with strategic } \\
\text { partners in an effort to systematically } \\
\text { improve OHCA response. } \\
\text { - DA-CPR or tele-CPR is where a caller to } \\
\text { the EMS system is asked to intervene and } \\
\text { is given instructions by the dispatcher. } \\
\text { Bystander CPR rate in Singapore } \\
\text { increased dramatically after DA-CPR } \\
\text { was implemented. The survival rate also } \\
\text { increased. }{ }^{\text {(26) }} \\
\text { - Alert apps are a good way to enable your } \\
\text { community to help. Smartphones are } \\
\text { common and can be leveraged to get } \\
\text { greater use and coverage by the app. } \\
\text { - Ambulance care is dependent on the } \\
\text { maturity of a particular EMS system. Its } \\
\text { development and the improvements that } \\
\text { can follow should not be overlooked. } \\
\text { - Infrastructure and traffic conditions also } \\
\text { play an important role, particularly in a } \\
\text { 'scoop and go' system. }\end{array}$ & $\begin{array}{l}\text { - Hospital-based care is } \\
\text { important, but this is only as } \\
\text { effective as the earlier parts } \\
\text { of the chain of survival. }{ }^{(6)} \\
\text { - Common interventions } \\
\text { include advanced } \\
\text { resuscitation rooms, } \\
24 / 7 \text { angiogram facilities, } \\
\text { hypothermia treatment and } \\
\text { post-resuscitation care. }\end{array}$ \\
\hline
\end{tabular}

AED: automated external defibrillator; CPR: cardiopulmonary resuscitation; DA-CPR: dispatcher-assisted cardiopulmonary resuscitation; EMS: emergency medical services

\section{Ongoing research}

The simultaneous implementation of the three community interventions (DA-CPR, CPR+AED training and myResponder mobile app) had positive cumulative effects on bystander CPR and was associated with increased survival to hospital discharge. ${ }^{(24)}$ To improve the effectiveness of bystander CPR and survival after OHCA, researchers have introduced the next-generation CPRcard, which will enable SCDF dispatchers to remotely observe the quality of CPR compressions being delivered to patients by myResponders in real time. Volunteers pair their next-generation CPRcard, which is Bluetooth-enabled, with their phone and resume their usual myResponder voluntary activities. This technological enhancement will enable SCDF dispatchers to not only observe the quality of compressions being delivered but also coach the responder based on what they observe. We believe that the enhanced CPRcard will result in better-quality chest compressions, and this will be the subject of our upcoming study.

\section{POLICY/PROTOCOL RECOMMENDATIONS}

Table I lists some community-based interventions that we have implemented over the years, which may be helpful to those who are considering a systematic approach to save lives. ${ }^{(25)}$

\section{DA-CPR protocol}

When calls come in for a suspected cardiac arrest, the caller is in the best position to render a life-saving intervention. However, many individuals are not trained in these skills or do not remember their training. Therefore, having a trained dispatcher provide 'justin-time' directions could be the determining factor for saving a life. The bystander CPR rate in Singapore increased dramatically after DA-CPR was implemented. Consequently, the patient survival rate also increased. ${ }^{(26)}$

\section{OHCA alert app (myResponder)}

Smartphone alert apps are important to speed up critical help for patients with OHCA. In most cases, trained and motivated responders in the vicinity can respond faster than EMS. Hence, this is a good intervention to pair with AED installations.

\section{AED installations}

An AED is life-saving for a victim of OHCA with a shockable heart rhythm, as it restores the heart rhythm to normal. This device is most effective during the initial moments of cardiac arrest. It is the hallmark of an advanced community response to OHCA to have in place a system of installed AEDs, and for citizens to retrieve and apply them to the victim before 
EMS arrives. ${ }^{(27,28)}$ Singapore now has nearly 8,000 installed AEDs, mostly in residential areas, where $70 \%$ of cardiac arrests occur. ${ }^{(29)}$ We have also set up a national Registry for Automated External Defibrillator Integration ${ }^{(29)}$ that feeds the myResponder App.

\section{Training schoolchildren}

Having a high degree of CPR/AED saturation in the populace is a good safeguard for sustaining successful improvements in the response to OHCA. Relevant knowledge and skills should be instilled starting from school-aged children. Studies show that schoolchildren are not too young to learn CPR and to be motivated to help others. ${ }^{(10,30)}$ We worked with the Ministry of Education, which has approved the teaching of CPR/AED skills in primary and secondary schools as part of the physical education curriculum. Teaching schoolchildren will help change cultural and public attitudes towards helping in a medical emergency. ${ }^{(10,30)}$

\section{Data registry for OHCA}

The PAROS registry was started using research funding. However, more permanent and sustainable support is required for data collection. A multi-ministry group has sought and obtained approval to set up a data pipeline for OHCA that will be hosted by the $\mathrm{MOH}$. Singapore has the luxury of a centralised EMS system, which provides us with national data on all OHCA cases that occur. This is a vital source of actionable data. Irrespective of whether an EMS system has a centralised system or a dispersed one, efforts should be made to gather data at the highest geographical level possible for a nation or locale.

\section{CONCLUSION}

A community-to-hospital systems approach is required to improve the response to $\mathrm{OHCA}$ because of the time-sensitive nature of the condition and its lethality. Treatment must be provided within the first five minutes of the onset of OHCA to improve the chance of survival in an otherwise healthy person. Although several factors work against the odds of survival, the interventions implemented in Singapore are designed to swing the odds in a favourable direction. Empowering and enabling the community, engaging the EMS system and bringing champions into the struggle are key elements in this approach. Enveloping all this effort with rigorous data collection lets the data show the way forward. Singapore's journey has been years in the making and, at times, progressed one step at a time, with many partners working collectively towards improvement. We are reaping its benefits now, and we need to continue our efforts in pursuit of even better quality and outcomes.

\section{SINGAPORE PAROS INVESTIGATORS}

Nausheen Edwin Doctor, Sengkang General Hospital, Singapore; Lai Peng Tham, KK Women's and Children's Hospital, Singapore; Han Nee Gan, Changi General Hospital, Singapore; Ling Tiah, Changi General Hospital, Singapore; Rabind Antony Charles, Department of Emergency Medicine, Woodlands Health Campus, Singapore; Si Oon Cheah, Urgent Care Clinic International, Singapore; Wei Ming Ng, Ng Teng Fong General Hospital,
Singapore; Wei Ling Tay, Ng Teng Fong General Hospital, Singapore; E Shaun Goh, Woodlands Health Campus, Singapore; Desmond Renhao Mao, Khoo Teck Puat Hospital, Singapore; Shir Lynn Lim, National University Heart Centre Singapore, Singapore; Gayathri D Nadarajan, Singapore General Hospital, Singapore; Ivan SY Chua, Singapore General Hospital, Singapore

\section{ACKNOWLEDGEMENTS}

We would like to thank the late Ms Susan Yap from the Department of Emergency Medicine, Singapore General Hospital; Ms Noor Azuin and Ms Joann Poh from the Unit for Prehospital Emergency Care (UPEC), Singapore General Hospital; Ms Charlene Ong from Accident and Emergency, Changi General Hospital; and Ms Woo Kai Lee from Department of Cardiology, National University Heart Centre Singapore for their contributions to the Singapore OHCA registry. We acknowledge Dr Lee Heow Yong, Ms Sarah Leong, Ms Geraldine Lee and their staff from the $\mathrm{MOH}$ (Hospital Services Division) for their support and fruitful collaboration; Mr Chiun How Teng (former Senior Manager, UPEC) and Ms Mayvin Yeow, Senior Manager, UPEC for their management and leadership; Ms Eileen $\mathrm{Ng}$, Ms Jinny Seow, Mr Chong Guan Seng, Ms Naomi John Lum and all training facilitators who helped to train tens of thousands of Singaporeans as part of the DARE team; and Laerdal Medical (Norway and Singapore offices) for their collaboration on the study involving the CPRcard. We are grateful to all the Medical Audit Specialists from UPEC, Singapore General Hospital for their support and efforts in implementing the Dispatcher-Assisted CPR programme and our colleagues from the SCDF, AC Yazid Abdullah, COL Bob Tan, MAJ Joey Ai Meng Tay, WO2 Pey Yun Low and CPT Carl Ross De Souza, for their fruitful collaboration. We would also like to thank Ms Patricia Tay from the Singapore Clinical Research Institute for her role as secretariat for the PAROS network.

\section{SOURCES OF FUNDING}

The PAROS study was supported by grants from the National Medical Research Council, Clinician Scientist Awards, Singapore (NMRC/CSA/024/2010,NMRC/CSA/0049/2013 and NMRC/CSASI/0014/2017) and Ministry of Health, Health Services Research Grant, Singapore (HSRG/0021/2012).

\section{DISCLOSURES}

Ong MEH reports funding from the Zoll Medical Corporation for a study involving mechanical CPR devices; grants from the Laerdal Foundation, Laerdal Medical and Ramsey Social Justice Foundation for funding of the Pan-Asian Resuscitation Outcomes Study; an advisory relationship with Global Healthcare SG, a commercial entity that manufactures cooling devices; and funding from Laerdal Medical on an observation programme to their Community CPR Training Centre Research Programme in Norway. Ong MEH has a licensing agreement and patent filed (Application no: 13/047,348) with ZOLL Medical Corporation for a study titled 'Method of predicting acute cardiopulmonary events and survivability of a patient'. All other authors have no conflict of interest to disclose. 


\section{REFERENCES}

1. Berdowski J, Berg RA, Tijssen JG, Koster RW. Global incidences of out-of-hospita cardiac arrest and survival rates: systematic review of 67 prospective studies. Resuscitation 2010; 81:1479-87.

2. Kiguchi T, Okubo M, Nishiyama C, et al. Out-of-hospital cardiac arrest across the world: first report from the International Liaison Committee on Resuscitation (ILCOR). Resuscitation 2020; 152:39-49.

3. Lai H, Choong CV, Fook-Chong S, et al. Interventional strategies associated with improvements in survival for out-of-hospital cardiac arrests in Singapore over 10 years. Resuscitation 2015; 89:155-61.

4. White AE, Poh JS, Pek PP, et al. Singapore out-of-hospital cardiac arrest registry report 2011-2018. In: Unit for Pre-hospital Emergency Care, Singapore. Available at: https://www.myheart.org.sg/wp-content/uploads/2021/01/ Singapore-OHCA-Data-Report-2011-2018.pdf. Accessed August 3, 2021.

5. Ong MEH, Chan $\mathrm{YH}$, Anantharaman $\mathrm{V}$, et al. Cardiac arrest and resuscitation epidemiology in Singapore (CARE I Study). Prehosp Emerg Care 2003; 7:427-33.

6. Cummins RO, Ornato JP, Thies WH, Pepe PE. Improving survival from sudden cardiac arrest: the "chain of survival" concept. A statement for health professionals from the Advanced Cardiac Life Support Subcommittee and the Emergency Cardiac Care Committee, American Heart Association. Circulation $1991 ; 83: 1832-47$

7. Ong MEH, Tan EH, Ng FSP, et al. Survival outcomes with the introduction of intravenous epinephrine in the management of out-of-hospital cardiac arrest. Ann Emerg Med 2007; 50:635-42.

8. Ong MEH, Shin SD, Tanaka H, et al. Pan-Asian Resuscitation Outcomes Study (PAROS): rationale, methodology, and implementation. Acad Emerg Med 2011; 18:890-7.

9. Ong MEH, Quah JLI, Ho AFW, et al. National population based survey on the prevalence of first aid, cardiopulmonary resuscitation and automated external defibrillator skills in Singapore. Resuscitation 2013; 84:1633-6.

10. Kua PHJ, White $A E, N g W Y$, et al. Knowledge and attitudes of Singapore schoolchildren learning cardiopulmonary resuscitation and automated external defibrillator skills. Singapore Med J 2018; 59:487-99.

11. Hallstrom A, Cobb L, Johnson E, Copass M. Cardiopulmonary resuscitation by chest compression alone or with mouth-to-mouth ventilation. N Engl J Med 2000; 342:1546-53.

12. Bobrow BJ, Spaite DW, Berg RA, et al. Chest compression-only CPR by lay rescuers and survival from out-of-hospital cardiac arrest. JAMA 2010; 304:1447-54

13. White $\mathrm{AE}, \mathrm{Ng} \mathrm{HX}, \mathrm{Ng} \mathrm{WY}$, et al. Measuring the effectiveness of a nove CPRcard $^{\mathrm{TM}}$ feedback device during simulated chest compressions by non healthcare workers. Singapore Med J 2017; 58:438-45.

14. Ng WM, De Souza CR, Pek PP, et al. myResponder smartphone application to crowdsource basic life support for out-of-hospital cardiac arrest: the Singapore experience. Prehosp Emerg Care 2021; 25:388-96.

15. Brooks SC, Simmons G, Worthington H, Bobrow BJ, Morrison LJ. The PulsePoint
Respond mobile device application to crowdsource basic life support for patients with out-of-hospital cardiac arrest: challenges for optimal implementation. Resuscitation 2016; 98:20-6.

16. Berglund E, Claesson A, Nordberg P, et al. A smartphone application for dispatch of lay responders to out-of-hospital cardiac arrests. Resuscitation 2018; 126:160-5.

17. Andelius L, Malta Hansen C, Lippert FK, et al. Smartphone activation of citizen responders to facilitate defibrillation in out-of-hospital cardiac arrest. J Am Coll Cardiol 2020; 76:43-53.

18. Anantharaman $\mathrm{V}, \mathrm{Ng} \mathrm{BLB}$, Ang $\mathrm{SH}$, et al. Prompt use of mechanical cardiopulmonary resuscitation in out-of-hospital cardiac arrest: the MECCA study report. Singapore Med J 2017; 58:424-31.

19. Ong MEH, Shin SD, Tanaka $\mathrm{H}$, et al. Rationale, methodology, and implementation of a dispatcher-assisted cardiopulmonary resuscitation trial in the Asia-Pacific (Pan-Asian Resuscitation Outcomes Study Phase 2). Prehosp Emerg Care 2015; 19:87-95.

20. Ho AFW, Sim ZJ, Shahidah N, et al. Barriers to dispatcher-assisted cardiopulmonary resuscitation in Singapore. Resuscitation 2016; 105:149-55.

21. Harjanto $S, N a M X B, H a o ~ Y$, et al. A before-after interventional trial of dispatcher-assisted cardio-pulmonary resuscitation for out-of-hospital cardiac arrests in Singapore. Resuscitation 2016; 102:85-93.

22. Nazeha N, Ong MEH, Limkakeng AT Jr, et al. A hypothetical implementation of 'Termination of Resuscitation' protocol for out-of-hospital cardiac arrest. Resuscitation Plus 2021; 6:100092.

23. Tan TX, Hao Y, Ho AF, et al. Inter-hospital variations in resuscitation processes and outcomes of out-of-hospital cardiac arrests in Singapore. J Emerg Crit Care Med 2019; 3:21.

24. Blewer AL, Ho AFW, Shahidah N, et al. Impact of bystander-focused public health interventions on cardiopulmonary resuscitation and survival: a cohort study. Lancet Public Health 2020; 5:e428-36.

25. Ong MEH, Perkins GD, Cariou A. Out-of-hospital cardiac arrest: prehospital management. Lancet 2018; 391:980-8.

26. Pek PP, Lim JYY, Leong BSH, et al. Improved out-of-hospital cardiac arrest survival with a comprehensive dispatcher-assisted CPR program in a developing emergency care system. Prehosp Emerg Care 2020 Dec 4. https://doi.org/.10. 1080/10903127.2020.1846824. [Epub ahead of print]

27. Rea T, Blackwood J, Damon S, Phelps R, Eisenberg M. A link between emergency dispatch and public access AEDs: potential implications for early defibrillation. Resuscitation 2011; 82:995-8.

28. Hallstrom AP, Ornato JP, Weisfeldt M, et al. Public-access defibrillation and survival after out-of-hospital cardiac arrest. N Engl J Med 2004; 351:637-46.

29. Chua SYI, Ng YY, Ong MEH. Getting R-AEDI to save lives in Singapore. Singapore Med J 2020; 61:60-2.

30. Kanstad BK, Nilsen SA, Fredriksen K. CPR knowledge and attitude to performing bystander CPR among secondary school students in Norway. Resuscitation $2011 ; 82: 1053-9$ 